\title{
Uma Formulação Hibridizada de Elementos Finitos para Problemas Parabólicos*
}

\author{
K.P. FERNANDES**, A.F.D. LOULA e S.C.M. MALTA
}

Recebido em 6 maio, 2013 / Aceito em 7 julho, 2013

\begin{abstract}
RESUMO. Este trabalho trata da aplicação de um método de elementos finitos descontínuo hibridizado, combinado com aproximações de diferenças finitas para a variável temporal, visando a solução de problemas parabólicos. Tal proposta foi desenhada considerando-se uma aproximação espacial descontínua entre os elementos, com a continuidade ao longo das interfaces imposta fracamente através do uso de um multiplicador de Lagrange. A precisão e eficiência da metodologia, quando comparada a aproximações espaciais usuais, por exemplo, o método de Galerkin contínuo, são comprovadas pelas taxas de convergência exibidas. Além disso, demonstra-se que é possível eliminar oscilações espúrias associadas a discretizações espaciais usualmente obtidas com formulações contínuas convencionais em problemas de condução de calor.
\end{abstract}

Palavras-chave: diferenças finitas, métodos híbridos, equação do calor, estabilização.

\section{INTRODUÇÃO}

Problemas parabólicos têm sido exaustivamente estudados sob os pontos de vista matemático e computacional. O método de Galerkin clássico, o qual é usualmente definido de maneira que a aproximação espacial seja contínua entre os elementos da discretização, é bastante empregado para resolver numericamente essa classe de problemas. Contudo, a aplicação desse método, quando combinado com aproximações por diferenças finitas na discretização da variável temporal, comumente denotado por metodologia semidiscreta usual, pode resultar no aparecimento de oscilações espúrias nos tempos iniciais [7].

Neste trabalho é proposto um método híbrido de elementos finitos, combinado com esquemas de diferenças finitas na integração temporal, visando resolver numericamente problemas parabólicos bi-dimensionais. Métodos híbridos de elementos finitos e de Galerkin Descontínuo (GD) [9] estão relacionados pelo uso de aproximações que utilizam funções localmente definidas (espaço de funções quebradas). Diferentemente dos métodos de GD, os métodos híbridos são

\footnotetext{
*Este trabalho foi parcialmente apresentado no CNMAC2012 - Águas de Lindóia, SP.

** Autor correspondente: Katia Fernandes

Laboratório Nacional de Computação Científica, LNCC/MCTI, Av. Getúlio Vargas, 333, 25651-075 Petrópolis, RJ, Brasil. E-mails: katiapf1@yahoo.com.br; aloc@lncc.br; smcm@lncc.br
} 
resolvidos em nível de elemento e as variáveis locais são eliminadas em favor do multiplicador de Lagrange, definido nas interfaces dos elementos. Consequentemente, o sistema global resultante é montado a partir dos graus de liberdade associados ao multiplicador de Lagrange, reduzindo assim o seu custo computacional e, principalmente, a sua complexidade computacional, tornandose semelhante aos métodos de elementos finitos contínuos. Será analisada a combinação de um método hibridizado de elementos finitos com esquemas explícitos, semi-implícitos e totalmente implícitos de diferenças finitas, aplicados na integração temporal. Essas opções serão comparadas entre si em relação ao custo computacional e a precisão (taxas de convergência). Finalmente, será mostrado que a formulação híbrida é apropriada para tratar as oscilações espúrias causadas por aproximações espaciais contínuas, como a metodologia semi-discreta acima mencionada.

Uma análise de erro completa, exibindo as propriedades de estabilidade, consistência, existência e unicidade de solução para o método híbrido aqui introduzido, assim como um estudo justificando os resultados obtidos em relação a sua robustez (eliminação das oscilações espúrias) serão tratadas em um trabalho futuro.

\section{A FORMULAÇÃ̃ VARIACIONAL HÍBRIDA}

Para introduzir a metodologia numérica proposta escolhe-se um problema parabólico clássico, que modela a condução de calor/temperatura $(u)$ em um domínio $\Omega \subset \mathbb{R}^{2}$, para um tempo $T>0$, descrito pelas seguintes equações:

Problema Global (PG): Encontrar $u: \Omega \times[0, T] \rightarrow \mathbb{R}$ tal que:

$$
\left\{\begin{aligned}
\frac{\partial u}{\partial t}-\Delta u & =f & & \operatorname{em~} \Omega \times[0, T] \\
u(x, y, t) & =0 & & \text { sobre } \partial \Omega \times[0, T] \\
u(x, y, 0) & =u_{0}(x, y) & & \operatorname{em~} \Omega,
\end{aligned}\right.
$$

com $f$ e $u_{0}(x, y)$ funções conhecidas, suficientemente regulares. Portanto, inicia-se com a noção de espaços das funções quebradas, os quais são usualmente utilizados nas definições de métodos de Galerkin Descontínuos [1, 2, 9]. Esses espaços dependem fortemente da partição/discretização do domínio, como será visto a seguir. Considera-se então $\mathcal{T}_{h}$ uma família regular de elementos $K$ compondo uma partição do domínio $\Omega \subset \mathbb{R}^{2}$, sendo $K$ um triângulo ou um quadrilátero (ou qualquer um outro tipo de forma). Denotando por $\partial K$ o conjunto das arestas do elemento $K$, seja $\mathcal{E}_{h}$ o conjunto das arestas/lados (inclusive nas fronteiras) de todos os elementos $K$ da partição/malha/família $\mathcal{T}_{h}$, onde $h$ é o diâmetro máximo do elemento. Além disso, seja $\mathcal{F}_{h}^{0}$ o conjunto das arestas internas da partição e $\mathcal{E}^{\partial}=\mathcal{E}_{h} \cap \partial \Omega$ o conjunto de todas as arestas de $\mathcal{E}_{h}$ sobre a fronteira de $\Omega$. Se $e$ é uma aresta compartilhada por dois elementos vizinhos $K_{1}$ e $K_{2}$ então, para uma função escalar $\varphi$ e uma função vetorial $\mathbf{v}$, suaves por partes em $\mathcal{T}_{h}$, definem-se médias e saltos, respectivamente, por

$$
\begin{array}{ll}
\{\varphi\}=\frac{1}{2}\left(\varphi^{1}+\varphi^{2}\right), \llbracket \varphi \rrbracket=\varphi^{1} \mathbf{n}^{1}+\varphi^{2} \mathbf{n}^{2} & \text { em } e \in \mathcal{E}_{h}^{0} ; \quad \text { e } \\
\{\mathbf{v}\}=\frac{1}{2}\left(\mathbf{v}^{1}+\mathbf{v}^{2}\right), \llbracket \mathbf{v} \rrbracket=\mathbf{v}^{1} \mathbf{n}^{1}+\mathbf{v}^{2} \mathbf{n}^{2} \quad \text { em } e \in \mathcal{E}_{h}^{0},
\end{array}
$$


com $\mathbf{n}^{1}$ e $\mathbf{n}^{2}$ os vetores normais unitários, apontados para o exterior dos elementos $K_{1}$ e $K_{2}$. Desse modo, define-se o problema local, em nível de cada elemento $K \in \mathcal{T}_{h}$, do seguinte modo:

Problema Local (PL): Para cada $t \in(0, T]$ e $x \in K$ encontrar $u(x, t)$, tal que:

$$
\begin{cases}\frac{\partial u}{\partial t}-\Delta u=f & \text { em } K \\ \llbracket \nabla u \rrbracket_{e}=0 & \forall e \in \mathcal{E}_{h}^{0} \\ \llbracket u \rrbracket_{e}=0 & \forall e \in \mathcal{E}_{h} \\ u(x, y, 0)=u_{0}(x, y) & \text { em } K .\end{cases}
$$

O problema (2.2) caracteriza o método proposto como sendo descontínuo entre os elementos $K$ da partição $\mathcal{T}_{h}$. É imediato verificar que os problemas (2.1) e (2.2) são equivalentes [2].

A seguir apresenta-se uma fomulação variacional híbrida para (2.2). Multiplicando (2.2) 1 por uma função $v \in V_{\left.\right|_{K}}$, onde $V=H^{2}\left(\mathcal{T}_{h}\right)=\left\{v \in L^{2}(\Omega),\left.v\right|_{K} \in H^{2}(K)\right\}$ e $V_{\left.\right|_{K}}=H^{2}(K)$, e integrando por partes no domínio $K$, obtém-se a forma fraca local:

$$
\left(\frac{\partial u}{\partial t}, v\right)_{K}+(\nabla u, \nabla v)_{K}-\int_{\partial K} \nabla u \cdot \mathbf{n} v d s=(f, v)_{K} .
$$

O último termo do lado esquerdo da equação (2.3) surge naturalmente da integração por partes e assegura a consistência do método. Seguindo as idéias de Nitsche [8] e Babuška [3] acrescenta-se a equação (2.3) um termo de simetrização e um termo de estabilização, que garantem, respectivamente, as propriedades de consistência adjunta e estabilidade. Deste modo, a identidade (2.3) é reescrita como:

$$
\begin{gathered}
\left(\frac{\partial u}{\partial t}, v\right)_{K}+(\nabla u, \nabla v)_{K}-\int_{\partial K} \nabla u \cdot \mathbf{n} v d s \\
-\int_{\partial K}(u-\lambda) \nabla v \cdot \mathbf{n} d s+\int_{\partial K} \beta(u-\lambda) v d s=(f, v)_{K}, \forall v \in V_{\left.\right|_{K}}
\end{gathered}
$$

sendo $\beta$ um parâmetro de estabilização, a ser definido posteriormente, e $\lambda$ o multiplicador de Lagrange, associado ao traço de $u$ nas arestas $e, \operatorname{com} \lambda \in M=\left\{\mu \in L^{2}(e), \forall e \in \mathcal{E}_{h}^{0}\right\}$. Logo, $\lambda$ é aqui tratado como uma variável independente, sendo calculado em nível de cada aresta.

Como o problema (2.4) contém as incógnitas $u$ e $\lambda$ para torná-lo possível de resolução acrescentase, ainda, a seguinte equação, associada ao multiplicador $\lambda$, resultante da imposição de forma fraca das condições de interface $\llbracket u \rrbracket_{e}=0$ e $\llbracket \nabla u \rrbracket_{e}=0$, a saber:

$$
\sum_{K}\left[\int_{\partial K} \nabla u \cdot \mathbf{n} \mu d s+\int_{\partial K} \beta(\lambda-u) \mu d s\right]=0, \quad \forall \mu \in M .
$$

Portanto, tem-se, associado ao problema local (PL), a seguinte

Formulação Variacional Híbrida (FVH1): Para cada $t \in(0, T]$, encontrar $u(t) \in V$ e $\lambda \in M$ satisfazendo as equações (2.4) e (2.5), $\operatorname{com} \beta=\beta_{0} / h, \beta_{0} \geq 0$, constante. 
Observa-se que FHV1 encontra-se no contexto das formulações hibridizadas pois a variável $u$ é calculada em nível (local) do elemento $K$, enquanto que o valor do multiplicador $\lambda$ obtém-se globalmente. É ainda conveniente escrever o problema fazendo uso da terminologia dos métodos de Galerkin Descontínuos (GD). Para isso, são levadas em conta as identidades apresentadas em Arnold et all [1], a equação (2.5), o fato que $u$ é a solução de (2.2) e $\lambda$ é descontínuo, resultando em uma nova expressão para a formulação variacional híbrida de PL, ou seja:

Formulação Variacional Híbrida (FVH2): Para cada $t \in(0, T]$, encontrar $u(t) \in V$ e $\lambda \in M$ tal que:

$$
\begin{gathered}
\sum_{K}\left\{\left(\frac{\partial u}{\partial t}, v\right)_{K}+(\nabla u, \nabla v)_{K}-\int_{\partial K} \nabla u \cdot \mathbf{n} v d s\right\} \\
-\sum_{K}\left\{-\int_{\partial K} \nabla v \cdot \mathbf{n}(u-\lambda) d s+\int_{\partial K} \beta(u-\lambda) v d s\right\}=\sum_{K}(f, v)_{K},
\end{gathered}
$$

para todo $v \in V$, onde $\lambda=\{u\}-\frac{1}{2 \beta} \llbracket \nabla u \rrbracket$.

\section{APROXIMAÇÕES POR DIFERENÇAS FINITAS NO TEMPO}

Nesta seção, são empregados os métodos de Euler explícito e implícito (diferenças finitas) na aproximação do termo da derivada temporal de FVH2 (e FVH1). Portanto, define-se uma partição do intervalo $[0, T]$ dada por $I=\left\{t_{0}=0, t_{1}, \cdots, t_{N}=T\right\}$, com $\Delta t=t_{n+1}-t_{n} \mathrm{o}$ tamanho do intervalo de tempo e denota-se $u\left(t^{n}\right)=u^{n}$.

Formulação Hibridizada Evolutiva (FHE): Para cada $K \in \mathcal{T}_{h}$ e $n=0, \ldots, N-1$, encontrar $\left(u^{n+1}, \lambda^{n+1}\right) \in V_{\left.\right|_{K}} \times M$, tal que:

$$
\left\{\begin{array}{l}
\left(\frac{u^{n+1}-u^{n}}{\Delta t}, v\right)_{K}+a\left(u^{n+1}, v\right)=f(v)-b\left(\lambda^{n}, v\right), \quad \forall v \in V_{\left.\right|_{K}} \\
\lambda_{\text {aux }}^{n+1}=\left\{u^{n+1}\right\}-\frac{1}{2 \beta} \llbracket \nabla u^{n+1} \rrbracket,
\end{array}\right.
$$

com as formas bilineares $a(u, v), b(\lambda, v)$ e a forma linear $f(v)$ dadas por:

$$
\begin{aligned}
a(u, v) & =(\nabla u, \nabla v)_{K}-\int_{\partial K} \nabla u \cdot \mathbf{n} v d s-\int_{\partial K} \nabla v \cdot \mathbf{n} v d s+\int_{\partial K} \beta u v d s, \\
b(\lambda, v) & =\int_{\partial K} \nabla v \cdot \mathbf{n} \lambda d s-\int_{\partial K} \beta \lambda v d s, \\
f(v) & =(f, v)_{K} .
\end{aligned}
$$

No cálculo do multiplicador de Lagrange $\lambda$ utiliza-se o método SOR (Successive Over Relaxation), ou seja, considera-se

$$
\lambda^{n+1}=(1-\omega) \lambda^{n}+\omega \lambda_{\text {aux }}^{n+1},
$$

sendo $\omega$ o parâmetro de relaxação, o qual acelera o processo de obtenção da solução aproximada. 
Visando melhorar a precisão da evolução temporal e a aproximação do multiplicador $\lambda$, foram propostos dois algoritmos iterativos, conforme descritos a seguir. $\mathrm{O}$ primeiro calcula, a cada passo de tempo $n+1, n=1, \ldots, N$, o termo de estabilização na iteração $k, k=1,2, \ldots$, da seguinte forma:

Formulação Hibridizada Penalti Semi-implícito (FHPSI): Para cada $K \in \mathcal{T}_{h}$ e $n=0, \ldots$, $N-1$, encontrar $\left(u^{n+1}, \lambda^{n+1}\right) \in V_{\left.\right|_{K}} \times M$, tal que:

$$
\left\{\begin{array}{l}
\left(\frac{u^{n+1, k+1}-u^{n}}{\Delta t}, v\right)_{K}+a\left(u^{n+1, k+1}, v\right)=f(v)-b\left(\lambda^{n+1, k}, v\right), \\
\lambda_{\text {aux }}^{n+1, k+1}=\left\{u^{n+1, k+1}\right\}-\frac{1}{2 \beta} \llbracket \nabla u^{n+1, k+1} \rrbracket
\end{array}\right.
$$

para todo $v \in V_{\left.\right|_{K}}$, com $\lambda^{n+1, k+1}=(1-\omega) \lambda^{n+1, k}+\omega \lambda_{\text {aux }}^{n+1, k+1}$. Considerando tol $>0$ um valor para a tolerância, a convergência do multiplicador é então verificada através de:

$$
\sum_{e \in \mathcal{F}_{h}^{0}} \frac{\sqrt{\left(\lambda_{e, \text { aux }}^{n+1, k+1}-\lambda_{e}^{n+1, k}\right)^{2}}}{\sqrt{\left(\lambda_{e, \text { aux }}^{n+1, k+1}\right)^{2}}} \leq \text { tol } \Rightarrow \begin{aligned}
& u^{n+1}=u^{n+1, k+1} \\
& \lambda^{n+1}=\lambda^{n+1, k+1} .
\end{aligned}
$$

No segundo algoritmo tem-se, no termo de estabilização, a temperatura $u$ e o multiplicador de Lagrange $\lambda$ obtidos de forma explícita. Desse modo, lê-se:

Formulação Hibridizada Penalti Explícito (FHPE): Para cada $K \in \mathcal{T}_{h}$ e $n=0, \ldots, N-1$, encontrar $\left(u^{n+1}, \lambda^{n+1}\right) \in V_{\left.\right|_{K}} \times M$, tal que:

$$
\left\{\begin{array}{l}
\left(\frac{u^{n+1, k+1}-u^{n}}{\Delta t}, v\right)_{K}+\tilde{a}\left(u^{n+1, k+1}, v\right)=f(v)-b\left(\lambda^{n+1, k}, v\right)-\int_{\partial K} \beta u^{n} v d s, \\
\lambda_{\text {aux }}^{n+1, k+1}=\left\{u^{n+1, k+1}\right\}-\frac{1}{2 \beta} \llbracket \nabla u^{n+1, k+1} \rrbracket,
\end{array}\right.
$$

para todo $v \in V_{\left.\right|_{K}}$ e a forma bilinear $\tilde{a}(u, v)$ definida por:

$$
\tilde{a}(u, v)=(\nabla u, \nabla v)_{K}-\int_{\partial K} \nabla u \cdot \mathbf{n} v d s-\int_{\partial K} \nabla v \cdot \mathbf{n} u d s .
$$

Um breve estudo comparativo entre as metodologias FHE, FHPSI e FHPE, com relação à variação dos parâmetros e eficiência, será apresentado na Seção 5, Resultados Computacionais.

Buscando ainda melhorar a precisão da evolução temporal e o custo computacional é agora proposta uma formulação totalmente implícita, isto é, uma aproximação em que as variáveis $u$ e $\lambda$ são calculadas na mesma iteração, sem a necessidade da utilização de um processo iterativo. Desse modo, define-se a 
Formulação Hibridizada Totalmente Implícita (FHTI): Para cada $K \in \mathcal{T}_{h}$ e $n=0, \ldots$, $N-1$, encontrar $\left(u^{n+1}, \lambda^{n+1}\right) \in V_{\left.\right|_{K}} \times M$, tal que:

$$
\left\{\begin{array}{l}
\left(u^{n+1}, v\right)_{K}+\Delta t\left[a\left(u^{n+1}, v\right)+b\left(\lambda^{n+1}, v\right)\right]=\Delta t f(v)+\left(u^{n}, v\right)_{K}, \quad \forall v \in V_{\left.\right|_{K}} \\
\sum_{K}\left[\int_{\partial K} \nabla u^{n+1} \cdot \mathbf{n} \mu d s+\int_{\partial K} \beta\left(\lambda^{n+1}-u^{n+1}\right) \mu d s\right]=0, \quad \forall \mu \in M .
\end{array}\right.
$$

Como será demonstrado nos experimentos numéricos da próxima seção, a FHTI apresenta melhor desempenho, sob os pontos de vista numérico e computacional, em relação as demais aproximações (FHPSI e FHPE), quando aplicada no cálculo da solução aproximada do problema de calor (2.1).

\section{FORMULAÇÃO HÍBRIDA COMPLETAMENTE DISCRETA}

Para a obtenção de uma aproximação totalmente discreta emprega-se o método de elementos finitos na discretização espacial das formulações introduzidas na seção anterior. Desse modo, são definidos os espaços de dimensão finita $V_{h}=\left\{v_{h} \in L^{2}(\Omega) ;\left.v_{h}\right|_{K} \in P_{l}(K), \forall K \in \mathcal{T}_{h}\right\}$ e $M_{h}=\left\{\mu_{h} \in L^{2}(e):\left.\mu_{h}\right|_{e} \in P_{m}(e), \forall e \in \mathcal{E}_{h}\right\}$, onde $P_{l}(K)$ e $P_{m}(K)$ são conjuntos de polinômios de grau $l$, se $K$ é um triângulo ou um quadrilátero, e de grau menor ou igual a $m$ sobre cada aresta/lado $e$, respectivamente. Para não tornar o texto repetitivo, apresenta-se, a seguir, apenas a forma totalmente discretizada da FHTI. As aproximações espaciais das outras formulações são obtidas de forma análoga.

Formulação Hibridizada Completamente Discreta (FHCD): Para cada $K \in \mathcal{T}$ e $n=0, \ldots$, $N-1$, encontrar $\left(u_{h}^{n+1}, \lambda_{h}^{n+1}\right) \in V_{h} \times M_{h}$ tal que:

$$
\left\{\begin{array}{l}
\left(u_{h}^{n+1}, v_{h}\right)_{K}+\Delta t\left[a\left(u_{h}^{n+1}, v_{h}\right)+b\left(\lambda_{h}^{n+1}, v_{h}\right)\right]=\Delta t f\left(v_{h}\right)+\left(u_{h}^{n}, v_{h}\right)_{K}, \\
\sum_{K}\left[\int_{\partial K} \nabla u_{h}^{n+1} \cdot \mathbf{n} \mu_{h} d s+\int_{\partial K} \beta\left(\lambda_{h}^{n+1}-u_{h}^{n+1}\right) \mu_{h} d s\right]=0,
\end{array}\right.
$$

para todo $v_{h} \in V_{h}$ e $\mu_{h} \in M_{h}$.

Observa-se que as formulações hibridizadas propostas aqui possuem a propriedade de conservação sobre cada elemento $K \in \mathcal{T}$, herdadas dos métodos de GD [9]. De fato, fixado o elemento $\bar{K} \in \mathcal{T}$, devido a descontinuidade entre os elementos da partição é possível escolher a função $v_{h} \in V_{h}$ tal que

$$
v_{h}= \begin{cases}1, & \text { em } \bar{K}, \\ 0, & \forall K \neq \bar{K},\end{cases}
$$

e, deste modo, chegar ao resultado esperado a partir da equação (4.1) $)_{1}$ tomando para isso $\beta=0$, ou seja, o termo adicional $\int_{\bar{K}} \beta\left(u_{h}^{n+1}-\lambda_{h}^{n+1}\right) d \bar{K}$ é uma massa puramente numérica que é zero se o valor do penalti for zero. Em geral, esta massa artificial pode ser exatamente calculada e 
extraída, se necessário [9]. Além disso, as formulações hibridizadas, como a FHCD, apresentam estabilidade independentes da escolha dos espaços de aproximações $\left(V_{h}\right.$ e $\left.M_{h}\right)$, tanto para o tipo do polinômio interpolante (polinômios de Lagrange, Legendre e outros) como para a ordem (grau) de interpolação dos mesmos, podendo também as malhas serem tomadas estruturadas ou não-estruturadas. Desse modo, notam-se importantes vantagens sobre alguns métodos usuais de elementos finitos tais como os métodos mistos clássicos [4, 5, 10], onde é exigido um comprometimento entre as escolhas dos espaços de aproximação para que sejam asseguradas unicidade e existência de solução.

Computacionalmente, a melhor opção para resolver o sistema (4.1) é calcular primeiro a equação (4.1) 1 , em nível de elemento, utilizando a técnica de condensação estática, para obter $u$ em termos de $\lambda$. Em seguida, substituíndo-se $u$ na equação $(4.1)_{2}$, resolve-se o sistema global (somando as contribuições dos elementos) para $\lambda$. Essa metodologia, típica de elementos finitos hibridizados, será então empregada nos experimentos numéricos da próxima seção.

\section{RESULTADOS COMPUTACIONAIS}

Nas simulações numéricas do problema parabólico (2.1), com condição de contorno (Dirichlet) homogênea e condição inicial $u_{0}(x, y)$, foram empregadas malhas uniformes compostas por quadriláteros com $4 \times 4=16,8 \times 8=64,16 \times 16=256$ e $32 \times 32=1024$ elementos, na discretização do domínio quadrado $\Omega=[0,1] \times[0,1]$. Utilizou-se interpolação lagrangiana bilinear para a temperatura $u$ e dois pontos de integração nas arestas para o multiplicador $\lambda$, correspondendo a uma aproximação linear.

\subsection{Precisão das Formulações Propostas}

Tomando $f(x, y)=\sin (\theta \pi x) \sin (\theta \pi y)$ no problema de calor (2.1) tem-se a condição inicial

$$
u_{0}(x, y)=\frac{\gamma}{2 \theta^{2} \pi^{2}} \sin (\theta \pi x) \sin (\theta \pi y)
$$

cuja solução analítica/exata é dada pela seguinte expressão

$$
u(x, y, t)=\left[\frac{1}{2 \theta^{2} \pi^{2}}+\left(\frac{\gamma-1}{2 \theta^{2} \pi^{2}}\right) e^{-2 \theta^{2} \pi^{2} t}\right] \sin (\theta \pi x) \sin (\theta \pi y) .
$$

Logo, considerando-se uma malha de $16 \times 16$ elementos, $\Delta t=10^{-4}$ e os parâmetros $\beta_{0}=6$, $\theta=1$ e $\gamma=0,001$ observa-se na Figura 1 para $\omega=1$ (esquerda) e $\omega=2$ (direita) (ver [6]) que a FHE fornece resultados estáveis porém imprecisos (dependem fortemente da escolha de $\omega$ ).

Por outro lado, na Figura 2, com $\beta_{0}=6$ e $\Delta t=10^{-4}$ e a solução (Exata) calculada para $\gamma=0,01$ e $\theta=1$, na figura da esquerda, concluí-se que a FHPSI recupera a precisão da evolução temporal. Além disso, na figura da direita, fixada uma malha com $16 \times 16$ elementos, independente da condição inicial verifica-se a convergência da formulação para a solução estacionária do problema de calor (2.1). Todos esses resultados foram também obtidos para a FHPSE. 


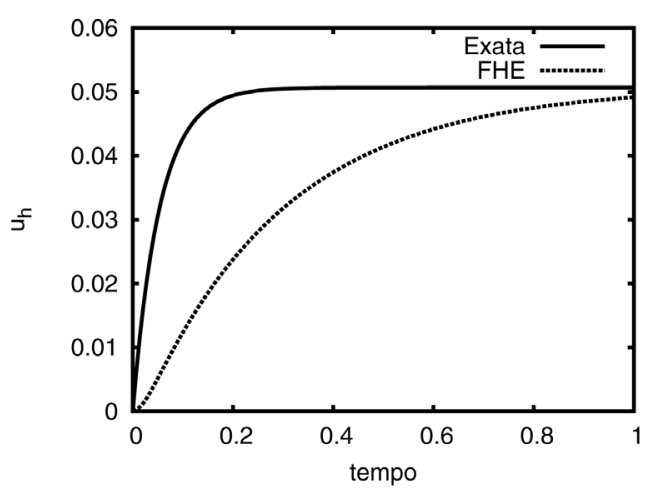

(a) $\omega=1$

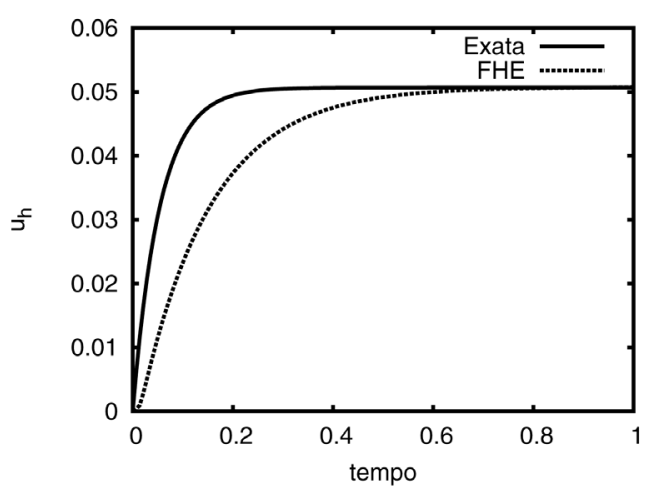

(b) $\omega=2$

Figura 1: Evolução temporal da temperatura $u_{h}$ obtida com a FHE no ponto central $(0,5 ; 0,5)$. Solução exata com $\gamma=0,001$ e $\theta=1$ em (a) $\omega=1$ e em (b) $\omega=2$.

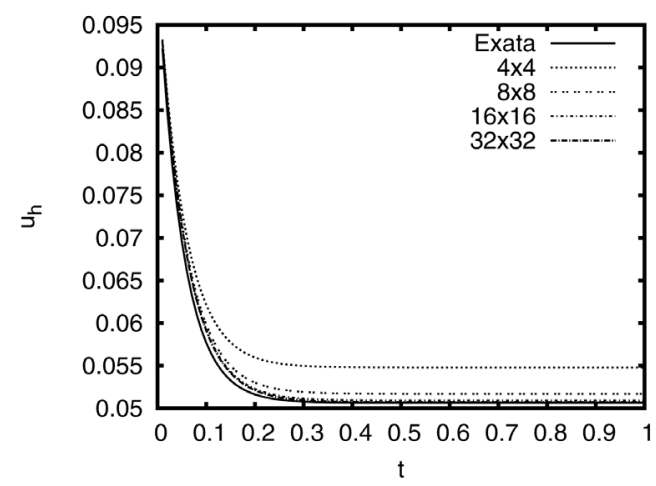

(a) diferentes malhas

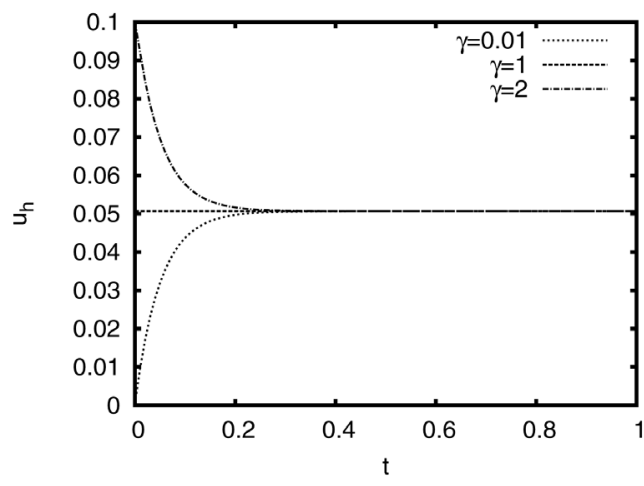

(b) diferentes condições iniciais

Figura 2: Evolução temporal da temperatura $u_{h}$ obtida com a FHPSI no ponto central $(0,5 ; 0,5)$. Em (a), Exata com $\gamma=0,01, \theta=1$ e diferentes malhas; em (b), malha $16 \times 16 \operatorname{com} \theta=1 \mathrm{e}$ diferentes valores de $\gamma$.

\subsection{Custo Computacional e Análise de Convergência}

Na Tabela 1 apresentam-se os tempos computacionais das metodologias propostas, considerando-se as três malhas $4 \times 4=16,8 \times 8=64$ e $16 \times 16=256$. Os dados adotados são $u_{0}(x, y)=\sin (\pi x) \sin (\pi y) / \pi^{2}(\gamma=2$ e $\theta=1$ na equação $(5.1)), \beta_{0}=12$, tol $=10^{-10}$, $\Delta t=10^{-6}$ e $n=150$ para a condição inicial de (2.1), o parâmetro de estabilização, a tolerância, o passo de tempo e o número de iterações, respectivamente. A convergência é alcançada em, no máximo, 6 iterações. Entre cada passo de tempo tem-se uma média de 4 iterações tanto para a FHPSI quanto para a FHPE. De acordo com os resultados da Tabela 1 conclui-se que a FHTI (ou FHCD) fornece os melhores resultados já que não há necessidade de iterações nessa formulação. Portanto, a FHTI (ou FHCD) será empregada na obtenção da solução aproximada $\left(u_{h}\right)$ nas simulações analisadas nesta seção. 
Tabela 1: Tempo computacional em segundos

\begin{tabular}{|c|c|c|c|}
\hline & \multicolumn{3}{|c|}{ Número de elementos } \\
\hline Método & $4 \times 4$ & $8 \times 8$ & $16 \times 16$ \\
\hline FHPSI & 0,278 & 1,423 & 5,711 \\
\hline FHPE & 0,282 & 1,407 & 5,074 \\
\hline FHTI & 0,199 & 0,796 & 3,027 \\
\hline
\end{tabular}

A seguir, considerando-se os dados numéricos do estudo anterior, é mostrado uma análise de convergência $h$. Na Figura 3 apresentam-se as taxas de convergência, na norma $L^{2}(\Omega)$, para a temperatura $\left(u_{h}\right)$, o gradiente $\left(\nabla u_{h}\right)$ e o multiplicador $\left(\lambda_{h}\right)$, em $t=1$. Em $(a)$ exibem-se os erros de aproximação e em $(b)$ os resultados para as interpolantes $\left(u_{I}\right.$ e $\left.\lambda_{I}\right)$. Nota-se que a solução aproximada, seu gradiente e o multiplicador fornecem taxas de convergência iguais as das suas interpolantes (taxas ótimas), i.e., $O\left(h^{2}\right), O\left(h^{1}\right)$ e $O\left(h^{1,5}\right)$, respectivamente. Além disso, na figura da direita, é possível verificar uma maior precisão para o multiplicador.

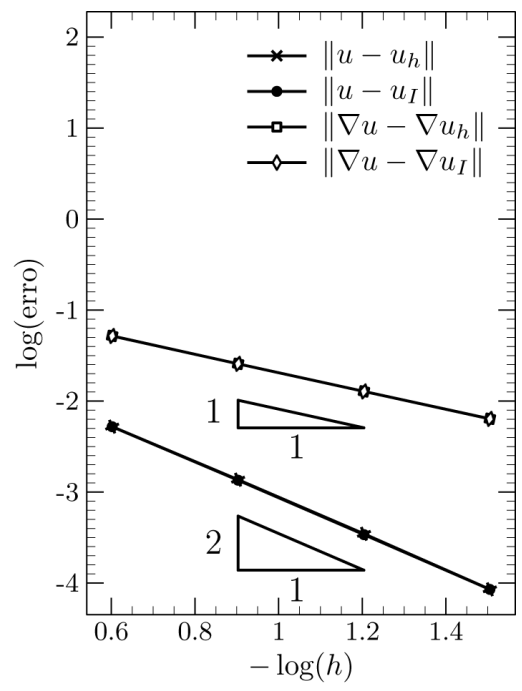

(a)

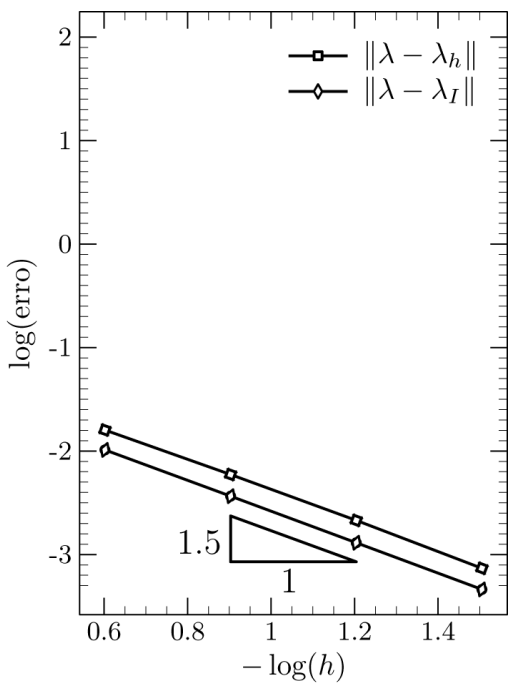

(b)

Figura 3: Estimativas de erro na norma $L^{2}(\Omega)$. Comparações entre as soluções aproximadas e suas interpolantes com $\beta_{0}=12$ e condição inicial $u_{0}(x, y)=\sin (\pi x) \sin (\pi y) / \pi^{2}$. Taxas de Convergência.

\subsection{Influência da Escolha do Parâmetro de Estabilização e Comparação com o Método de Galerkin Contínuo}

Inicialmente, nas simulações computacionais desta sub-seção, é analisada a influência do parâmetro de estabilização $\beta_{0}$ na resolução da equação do calor (2.1) para diferentes tipos de condições iniciais. 


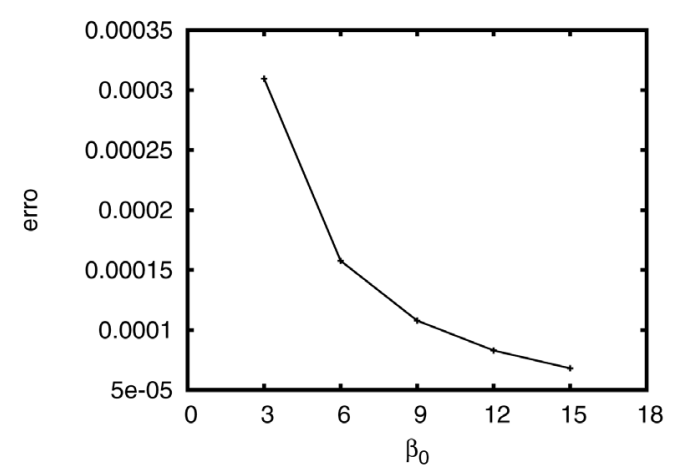

(a) $u_{0}(x, y)=\sin (\pi x) \sin (\pi y) / \pi^{2}(\theta=1)$

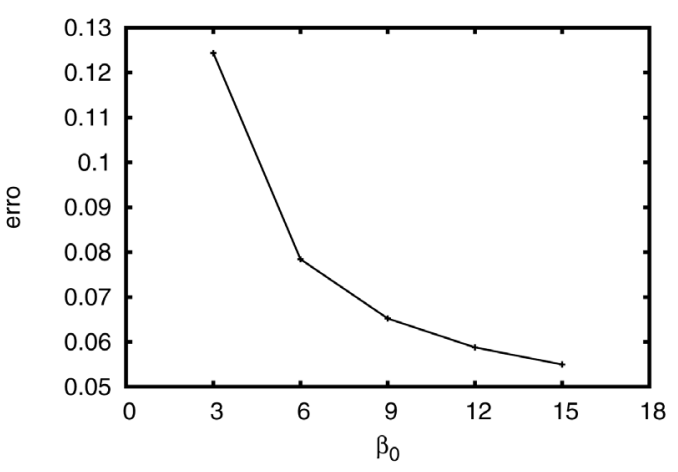

(b) $u_{0}(x, y)=\sin (\pi x) \sin (\pi y) / 14 \pi^{2}(\theta=7)$

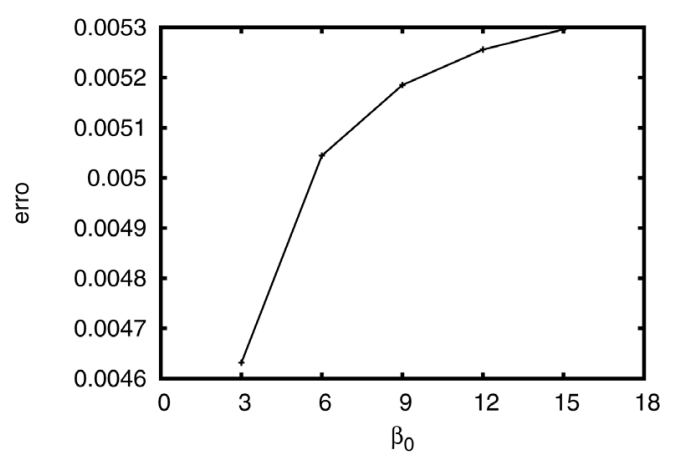

(c) $u_{0}(x, y)=1$

Figura 4: Erro relativo com $\beta_{0}=3,6,9,12,15$, malha $32 \times 32$ e $\Delta t=10^{-6}$, no ponto central $(0,5 ; 0,5)$ em $t=0,01$.

Na Figura 4 é exibido um estudo do comportamento de $\beta_{0}$ em relação ao erro relativo supondose dados regulares e não-regulares para uma malha com $32 \times 32$ elementos, um passo de tempo $\Delta t=10^{-6}$ e $\beta_{0}=3,6,9,12,15 \mathrm{em} t=0,01$. Em (a) e (b) o erro relativo é calculado entre a solução exata (5.2), com $u_{0}(x, y)=\gamma \sin (\pi x) \sin (\pi y) / 2 \theta^{2} \pi^{2}(\gamma=2, \theta=1$ e $\theta=7) \mathrm{e}$ a solução aproximada $\left(u_{h}\right)$. Por outro lado, em (c), considera-se uma condição inicial menos regular, $u_{0}(x, y)=1$. Então o erro é dado pela diferença entre uma solução de referência, obtida pela aplicação da metodologia semidiscreta em uma malha mais refinada ( $64 \times 64$ elementos), e a solução aproximada. Quando $\beta_{0}$ cresce observa-se um comportamento completamente distinto para o erro. A condição inicial suave (figuras (a) e (b)), mesmo com modos altos $(\theta=7)$, leva ao decrescimento e a uma escolha ótima $\beta_{0}=15$. Porém, com o dado não regular (figura (c)) tem-se o crescimento do erro, sendo $\beta_{0}=3$ a melhor opção. Uma investigação mais profunda sobre esse comportamento será tratada em um trabalho futuro.

No trabalho de I. Harari [7] foi mostrado que a solução de problemas parabólicos, com dados não regulares, obtidas através da metodologia semidiscreta (o método de Galerkin é aplicado na aproximação espacial e combinado com a aproximação de Euler implícito na variável temporal) pode resultar no aparecimento de oscilações espúrias nos tempos iniciais de simulação. 


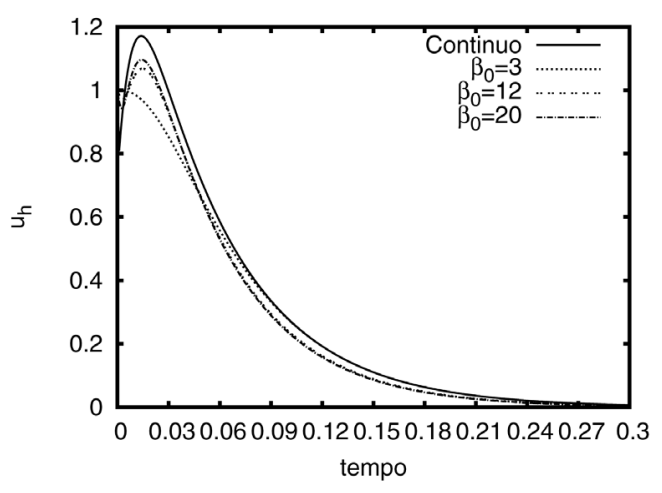

(a) Malha $4 \times 4$

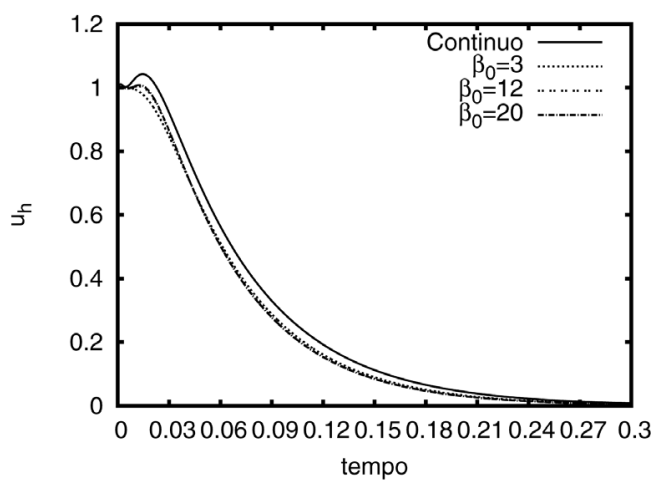

(c) Malha $8 \times 8$

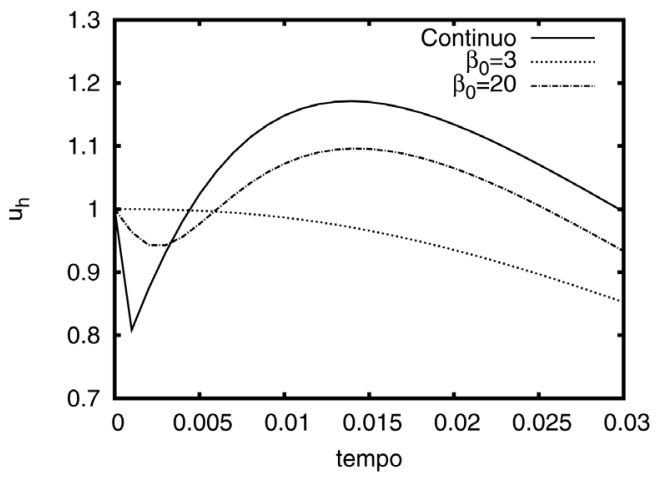

(b) Zoom malha $4 \times 4$

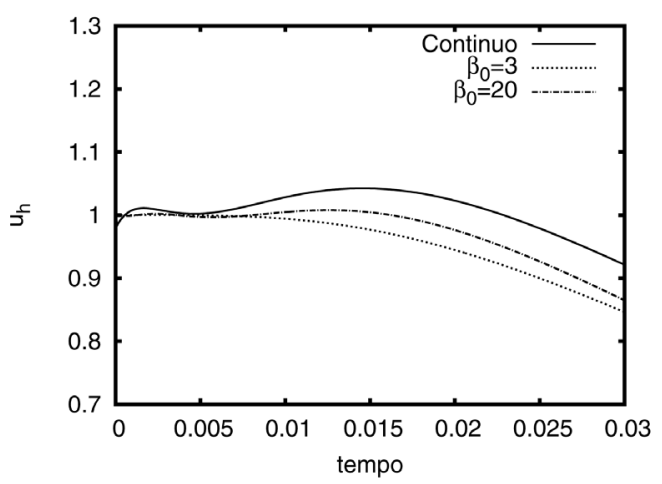

(d) Zoom malha $8 \times 8$

Figura 5: Evolução temporal da temperatura $u_{h}$ para diferentes valores de $\beta_{0}$, no ponto central $(0,5 ; 0,5)$. Comparação entre a metodologia semidiscreta (Contínuo) e a FHCD. Em (a) e (c), malhas $4 \times 4$ e $8 \times 8$, com $\Delta t=10^{-3}$ e $\Delta t=10^{-4}$, respectivamente. Em (b) e (d), ampliações dos resultados das figuras (a) e (c) para os instantes iniciais.

Desse modo, estuda-se também aqui a eficiência da FHCD quando comparada a tal metodologia considerando-se $u_{0}(x, y)=1$ e diferentes valores de $\beta_{0}$. Na Figura 5 tem-se o histórico no tempo da temperatura $u_{h}$, no ponto central do domínio. Em (a) e (b), adota-se uma malha $4 \times 4$ e $\Delta t=10^{-3}$. Em (c) e (d) utiliza-se uma malha $8 \times 8$ e $\Delta t=10^{-4}$.

Assim como no estudo exibido na Figura 4, é imediato observar a influência do valor do termo de estabilização nos resultados apresentados. Nota-se que para $\beta_{0}=9$ e 20 (grande) tem-se a presença das oscilações espúrias encontradas também com a metodologia semidiscreta (Continuo). Essas deixam de existir quando escolhe-se $\beta_{0}=3$. Desse modo, nota-se que à medida que $\beta_{0}$ cresce as aproximações obtidas com a formulação híbrida (descontínua) aproximam-se daquelas calculadas com a metodologia semidiscreta (Continuo), ou seja, as oscilações crescem com o aumento da constante $\beta_{0}$, do termo de estabilização. Esses comportamentos podem ser melhor visualizados nas figuras a direita, onde são apresentadas ampliações das simulações para os instantes iniciais. 
Dando continuidade a análise do problema estudado nesta sub-seção, para uma melhor visualização das oscilações, são exibidos na Figura 6 os resultados plotados na Fig. 5(c) para $\beta_{0}=3$ (escolha ótima). Como anteriormente, nota-se que a formulação hibridizada (figuras da direita) é capaz de eliminar as oscilações espúrias que surgem nos tempos iniciais $(n=2$ e $n=6$ ) na solução aproximada obtida pela metodologia semidiscreta (figuras da esquerda). Com a evolucão temporal ( $n=50$, último gráfico a esquerda) ambas soluções já exibem o mesmo comportamento.
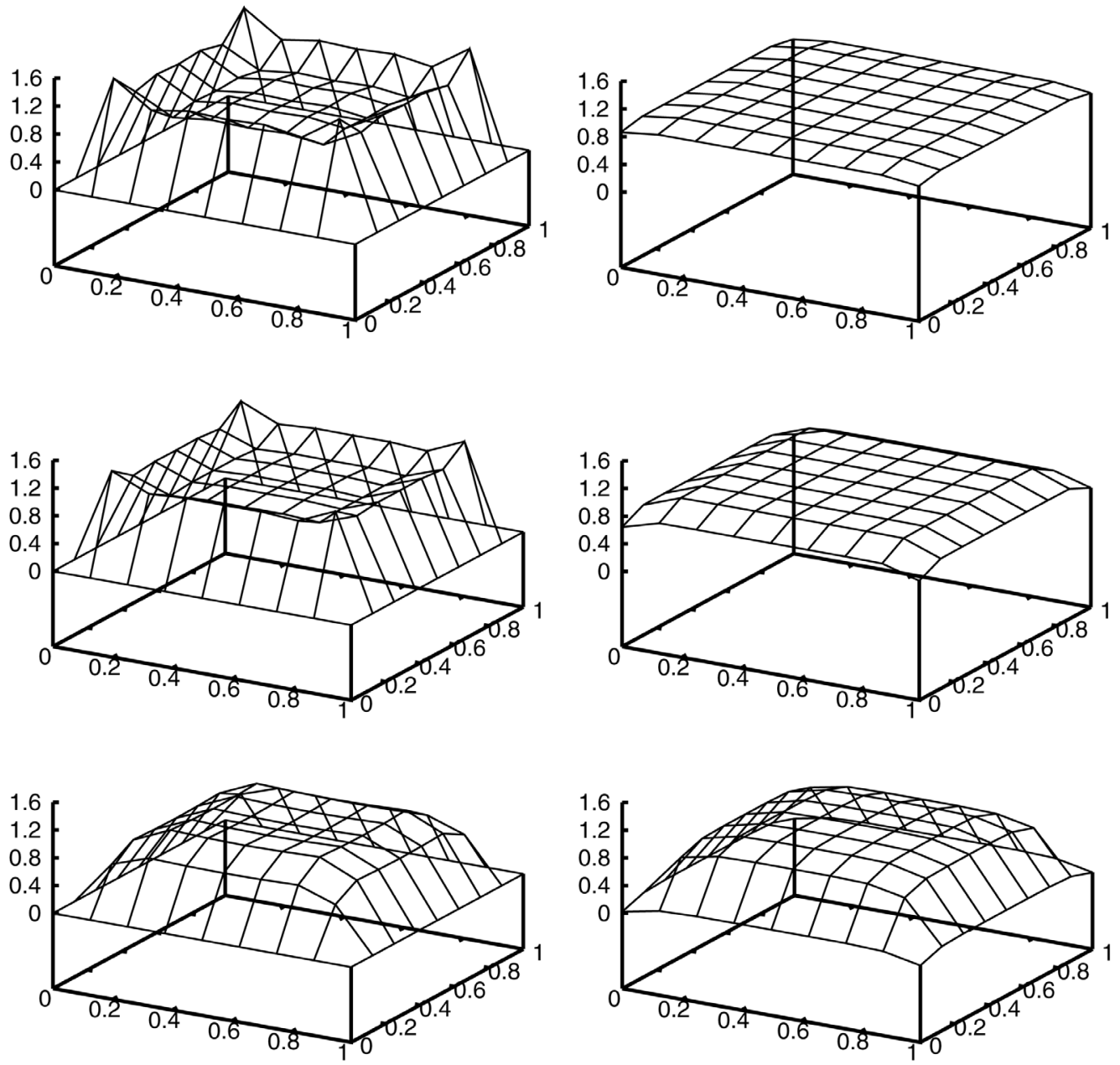

Figura 6: Evolução temporal da temperatura $u_{h}$ no ponto central $(0,5 ; 0,5)$. Comparações entre o Método de Galerkin Contínuo (esquerda) e a FHCD (direita) com malha $8 \times 8, \Delta t=10^{-4} \mathrm{e}$ $\beta_{0}=3$ para $n=2$, 6 e 50 , de cima para baixo.

Com base nos resultados encontrados pode-se concluir que a formulação hibridizada proposta fornece soluções que não são poluidas pelo erro na aproximação espacial, como acontece quando 
é empregado o método de Galerkin combinado com algum método da família trapezoidal na aproximação temporal de problemas parabólicos (veja [7] e os trabalhos citados).

\title{
6 CONCLUSÃO
}

Neste trabalho foram analisadas combinações de um método hibridizado de elementos finitos com esquemas explícitos (FHPE), semi-implícitos (FHPSI) e totalmente implícitos (FHTI) de diferenças finitas, empregados na integração temporal, visando a solução numérica de problemas parabólicos, em especial da equação do calor bi-dimensional. Observou-se, ainda, que a FHTI apresenta um custo computacional reduzido se comparado com as demais aproximações. Além disso, para condições iniciais não-regulares, quando o parâmetro de estabilização $\beta_{0}$ cresce a FHTI aproxima-se de uma formulação de Galerkin contínuo; contudo, essa abordagem não é interessante devido ao aparecimento de oscilações espúrias. Ao reduzirmos os valores de $\beta_{0}$ encontrou-se um $\beta_{0}$ ótimo, $\beta_{0}=3$, livre das oscilações.

Em trabalhos futuros pretende-se apresentar uma análise numérica que justifique a eliminação das oscilações espúrias, presentes no método de Galerkin contínuo, quando empregada a FHTI. Planeja-se, também, estender a aplicação deste estudo na solução de problemas transientes de difusão e reação.

\section{AGRADECIMENTOS}

Os autores agradecem ao Programa PCI/LNCC/MCTI e CNPq, projetos nos. 352991/1992-5 e 304764/2011-6, respectivamente. Os autores também agradecem as contribuições dos árbitros anônimos, que em muito melhoraram a versão deste trabalho.

\begin{abstract}
To solve parabolic problems a hybrid finite element method (HFEM) is introduced to numerically solve the bi-dimensional heat problem combined with finite difference time integration schemes. Such a formulation has been designed considering discontinuous spatial discretizations with continuity along the interface imposed via a Lagrange multiplier. The accuracy and efficiency of the proposed hybrid method are compared against the standard Galerkin method. Furthermore, the HFEM is applied to treat the spurious spatial oscillation problems commonly present in the continuous Galerkin semi-discrete approaches when applied to parabolic problems. For an appropriate choice of the stabilization parameter, numerical simulations will show that the HFEM is well suited to remediate these pathologies.
\end{abstract}

Keywords: finite differences, hybrid methods, heat equation, stabilization.

\section{REFERÊNCIAS}

[1] D.N. Arnold, F. Brezzi, B. Cockburn \& L.D. Marini. Unified analysis of discontinuous Galerkin methods for elliptic problems. SIAM J. Numer. Anal., 39 (2002), 1749-1779. 
[2] F. Brezzi, B. Cockburn, L.D. Marini \& E. Süli. Stabilization mechanisms in discontinuous Galerkin methods. Comput. Methods Appl. Mech. Engrg., 195 (2006), 3293-3310.

[3] I. Babuška. The finite element method with penalty. Math. Comp., 27 (1973), 221-228.

[4] H. Egger \& J. Schoberl. A hybrid mixed discontinuous Galerkin finite-element method for convection-diffusion problems. IMA Journal of Numerical Analysis, 30 (2010), 1206-1234.

[5] M. Farhloul \& M. Fortin. Review and complements on mixed-hybrid finite element methods for fluid flows. J. Comput. Appl. Math., 140 (2002), 301-313.

[6] K.P. Fernandes, S.M.C. Malta \& A.F.D. Loula. Formulações de elementos finitos hibridizadas para a equação do calor. In: Anais do XXXIV CNMAC, Águas de Lindóia, setembro, SP, Vol. IV, (2012).

[7] I. Harari. Stability of semidiscrete formulations for parabolic problems at small time steps. Comput. Methods Appl. Mech. Engrg., 193 (2004), 1491-1516.

[8] J. Nitsche. Uber ein variationsprinzip zur losung von dirichlet-problemen bei verwendung von teilraumen, die keinen randbedingungen unterworfen sind. Math. Sem. Univ. Hamburg, 36 (1971), 9-15.

[9] B. Riviére. "Discontinuous Galerkin Methods for Solving Eliptic and Parabolic Equations: Theory and Implementation", Frontiers in Applied Mathematics, SIAM Publication, New York (2008).

[10] J.E. Roberts \& J.M. Thomas. Mixed and hybrid methods, em "Finite Elements Methods (Part 1), volume II” (P.G. Ciarlet and J.L. Lions, eds.), Amsterdam, (1989), 523-639.

[11] V. Thomée. Galerkin Finite Element Methods for Parabolic Problems, Second Edition, "Springer Series in Computational Mathematics", Spring Verlag, The Netherlands (2006). 